\title{
Chronic Kidney Disease Is Associated with Increased Plasma Levels of Fibroblast Growth Factors 19 and 21
}

\author{
Małgorzata Marchelek-Myśliwiec ${ }^{a} \quad$ Violetta Dziedziejkob \\ Monika Nowosiad-Magdac Katarzyna Dołęgowska ${ }^{\mathrm{a}}$ \\ Barbara Dołęgowskac Andrzej Pawlik ${ }^{d}$ Krzysztof Safranow ${ }^{b}$ \\ Magda Wiśniewska ${ }^{a}$ Joanna Stępniewska ${ }^{a}$ Maciej Domańskia \\ Kazimierz Ciechanowskia \\ ${ }^{a}$ Clinical Department of Nephrology, Transplantology and Internal Medicine, Pomeranian \\ Medical University, Szczecin, Poland; 'b Department of Biochemistry and Medical Chemistry, \\ Pomeranian Medical University, Szczecin, Poland; ' $D$ epartment of Microbiology, \\ Immunology and Laboratory Medicine, Pomeranian Medical University, Szczecin, Poland; \\ ${ }^{\mathrm{d}}$ Department of Physiology, Pomeranian Medical University, Szczecin, Poland
}

\section{Keywords}

Adipose tissue - Body composition - Chronic kidney disease · Haemodialysis - Fibroblast growth factor $19 \cdot$ Fibroblast growth factor $21 \cdot$ Kidney transplantation

\begin{abstract}
Background: Chronic kidney disease (CKD) is the result of a reduced number of nephrons, in which adipose tissue and its metabolites play a significant role. Fibroblast growth factors, FGF19 and FGF21, are involved in lipid and carbohydrate metabolism. The aim of the study was to examine the concentrations of FGF19 and FGF21 in patients with CKD, as well as the correlation between FGF19 and FGF21 and selected biochemical parameters. Materials and Methods: The study included 178 subjects: 52 patients with CKD in stages 2-4, without haemodialysis (CKD), 47 haemodialysed patients with CKD (HD), 56 patients with CKD after a renal transplantation (Tx) and 23 healthy subjects as the control group (C). Results: The highest FGF19 serum concentrations were observed in CKD patients and the lowest were observed in the Tx group. Patients in the CKD group had significantly higher serum FGF21 concentrations. There were negative correlations between FGF19 and glomerular filtration rate (GFR), as well as high-density lipoprotein cholesterol levels in patients after kidney transplantation. Negative correlations were also found between serum FGF21 concentrations and GFR in patients after Tx, while positive correlations were observed between FGF21 concentrations and lean
\end{abstract}




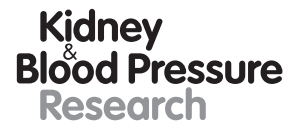

Kidney
Blood Pressure
Research \begin{tabular}{l|l}
\hline Kidney Blood Press Res 2019;44:1207-1218 \\
\hline DOI: 10.1159/000502647 & $\begin{array}{l}\text { @ 2019 The Author(s). Published by S. Karger AG, Basel } \\
\text { www.karger.com/kbr }\end{array}$ \\
\hline
\end{tabular}

Marchelek-Myśliwiec et al.: FGF in CKD

body mass in the CKD group, body mass index and total cholesterol in the HD group. Conclusions: Our results suggest that increased concentrations of FGF19 and FGF21 in patients with CKD may be associated with the metabolism of lipids and carbohydrates. Our results also indicate that haemodialysis and transplantation results in the reduction of FGF19 and FGF21 concentrations in patients with CKD.

(C) 2019 The Author(s)

Published by S. Karger AG, Basel

\section{Introduction}

Chronic kidney disease (CKD) manifests as a group of symptoms resulting from a reduction in the number and function of active nephrons. Because kidneys play an important role in maintaining homeostasis of the water-electrolyte, ionic (including calcium-phosphate) and haematopoietic systems, kidney damage negatively affects many metabolic pathways. CKD is associated with bone osteodystrophy, rapid progression of atherosclerosis and anaemia [1]. The pathophysiology of these disorders is relatively well understood, with recent studies indicating a significant role of adipose tissue and its metabolites in the course and progression of CKD [2].

Adipose tissue and its relevance in the prognosis of patients with CKD are the subject of intensive research. In the general population, obesity is an independent risk factor for the development of end-stage renal disease (ESRD) [3]. However, pre-dialysis, in CKD patients in more advanced disease stages, a higher body mass index (BMI) was not associated with an increased mortality rate [4]. In haemodialysed (HD) patients, studies have shown that a higher content of fat tissue may have a protective effect, and individuals with a low BMI and low body fat have a worse survival prognosis [5]. In patients after kidney transplantation, obesity affects the short-term transplant results and the development of glucose intolerance and diabetes [6].

White adipose tissue also plays a significant role in the development of metabolic disturbances (insulin resistance, dyslipidaemia and chronic low-grade inflammation), which are a common feature in patients with CKD [7]. Such disturbances are associated with increased mortality and morbidity in these patients [8]. Endocrine fibroblast growth factors (FGFs) provide beneficial effects for lipid and carbohydrate metabolism. Their concentrations change in chronic disorders, such as diabetes, obesity, metabolic syndrome and cardiovascular and renal diseases. FGFs improve glucose tolerance, insulin sensitivity, weight and lipid loss, as well as energy metabolism [9].

Recent studies have focused on the relatively recently described FGFs FGF19 and FGF21. These 2 growth factors belong to the FGF19 subfamily (FGF19, FGF21 and FGF23), and their activity is dependent on the presence of the cofactor, Klotho protein. FGF19 and FGF21 are secreted into the blood where they act as hormones via FGFR receptors [10]. Their clinical significance and their role in CKD are not yet fully understood; however, recent animal studies have revealed their beneficial metabolic effects in kidney disease [11].

FGF23 is produced in osteocytes and osteoblasts. In the kidneys, FGF23 regulates renal phosphate, sodium and calcium reabsorption and decreases $1.25(\mathrm{OH}) 2 \mathrm{D}$ synthesis. Moreover, in the parathyroid, FGF23 decreases parathyroid hormone gene expression and parathyroid cell proliferation [12].

FGF21 is primarily secreted by the liver and adipose tissue. It is synthesised in starvation and mediates, among others, lipolysis, thermogenesis, gluconeogenesis and fatty acid oxidation [13]. Elevated FGF21 levels are found in type 2 diabetes, obesity, metabolic syndrome and other disorders associated with insulin resistance [14, 15]. 


\section{Kidney \\ Blood Pressure \\ Research}

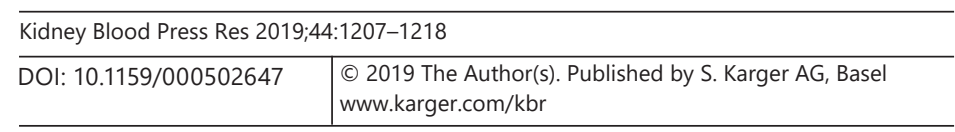

Marchelek-Myśliwiec et al.: FGF in CKD

FGF19 can be found primarily in the small intestine, gallbladder, brain and kidneys [9]. It inhibits bile acid synthesis. However, recent studies also suggest that FGF19 participates in carbohydrate metabolism as it stimulates glycogen synthesis and inhibits gluconeogenesis $[16,17]$. The role of FGF19 and FGF21 in CKD is unknown.

The aim of our study was to examine FGF19 and FGF21 concentrations in patients with CKD who were not HD, in HD patients with CKD and in patients with CKD after renal transplantation (Tx). We also examined the correlation between FGF19 and FGF21 and selected biochemical parameters of lipid and carbohydrate metabolism.

\section{Materials and Methods}

\section{Study Groups}

The study included 178 subjects: 52 patients with CKD in stages 2-4 (CKD) without haemodialysis, 47 HD patients with CKD, 56 patients with CKD after a Tx and 23 healthy subjects as the control group (C). The baseline characteristics of the study groups are shown in Table 1.

The inclusion criteria for the control group were as follows: over 18 years of age, no chronic inflammatory diseases (systemic lupus erythematosus, rheumatoid arthritis), no diabetes and glomerular filtration rate (GFR) above $60 \mathrm{~mL} / \mathrm{min} / 1.73 \mathrm{~m}^{2}$.

The following inclusion criteria were applied for the CKD group: over 18 years of age, no need for haemodialysis or renal replacement therapy and stable renal function for 3 months before enrolment. Exclusion criteria for this group were cancer and/or treatment with steroids at a dose of $>5 \mathrm{mg} /$ day. The causes of kidney insufficiency were as follows: diabetes $(D M)=12$, hypertension $(H A)=3$, autosomal dominant polycystic kidney disease $(A D P K D)=$ 3 , glomerulonephritis $(\mathrm{GN})=18$, other $=10$, congenital urinary tract malformations $(\mathrm{CD})=4$ and nephrolithiasis $(K S)=2$. The distribution of the CKD stages was (CKD 4) $=21$ patients, $($ CKD 3$)=28$ patients and (CKD 2) $=3$ patients. The number of (CKD 2) patients was too low for a separate analysis, so FGF21 and FGF19 concentrations were compared between (CKD $2+3$ ) patients (GFR $\geq 30$ ) and (CKD 4) patients (GFR $<30$ ).

Inclusion criteria for HD patients were over 18 years of age, haemodialysis therapy at least 12 months before enrolment, a dialysis period not longer than 5 years, a constant haemodialysis dose for at least 3 months before enrolment and a Kt/V above 1.2. Patients with a neoplastic disease were excluded. The causes of kidney insufficiency were as follows: DM = 9, $\mathrm{HA}=8, \mathrm{ADPKD}=2, \mathrm{GN}=7$ and other $=21$.

Inclusion criteria for transplant patients were Tx at least 2 years before enrolment, constant doses of immunosuppressive drugs for the last 3 months and standard immunosuppression with calcineurin inhibitor, mycophenolate mofetil and glucocorticosteroids. Exclusion criteria were neoplastic disease and/or bariatric surgery. The causes of kidney insufficiency were as follows: $\mathrm{DM}=5, \mathrm{HA}=11, \mathrm{ADPKD}=6, \mathrm{GN}=16$ and other $=18$.

This study was approved by the Bioethical Commission at the Pomeranian Medical University. All participants provided written informed consent.

\section{Material}

Blood samples in the C, CKD and Tx groups were collected from the peripheral vein (using Sarstedt S-Monovette tubes with clotting activator) after an overnight fast. In the HD patients, blood samples were collected before a haemodialysis procedure, after a 1-day interval between haemodialysis. Whole blood was centrifuged, and the serum samples were frozen at $-80^{\circ} \mathrm{C}$ until use. 
Kidney

Blood Pressure

Research
Kidney Blood Press Res 2019;44:1207-1218

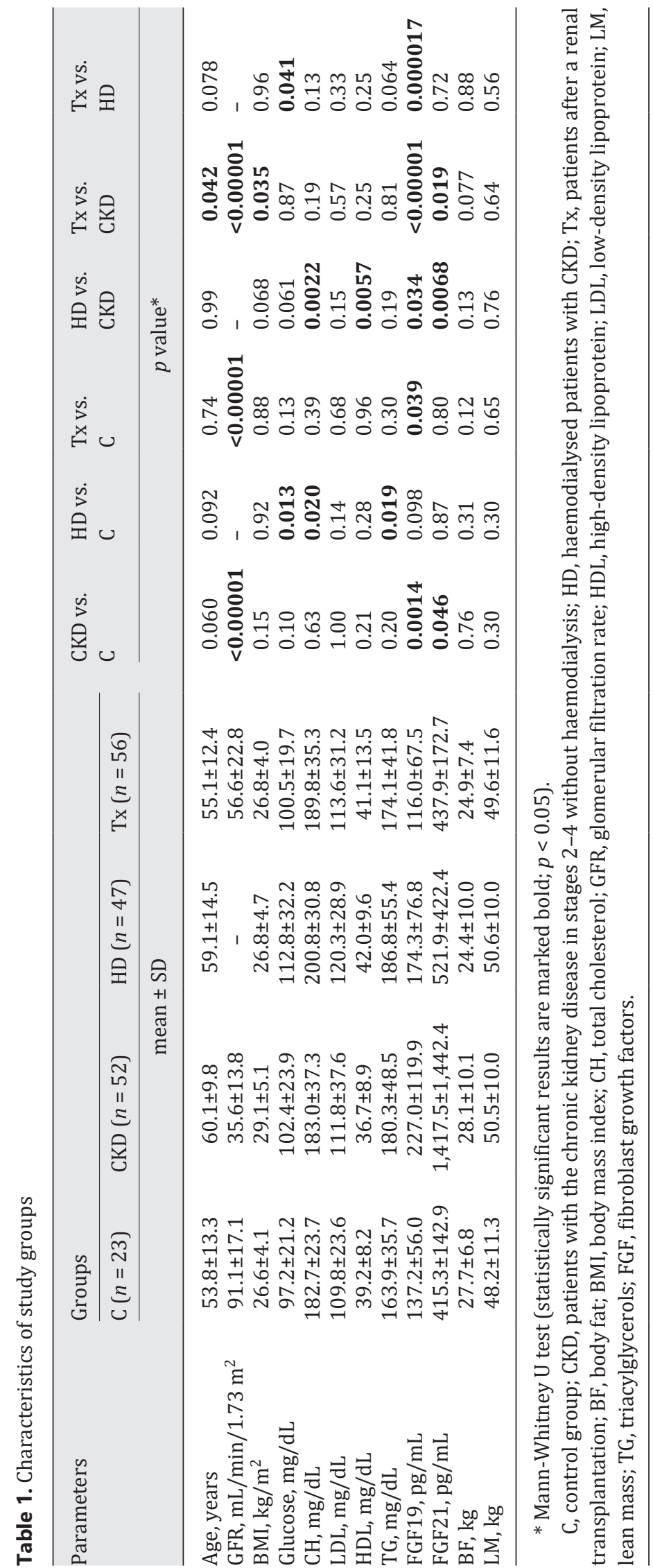




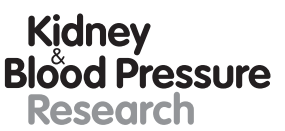

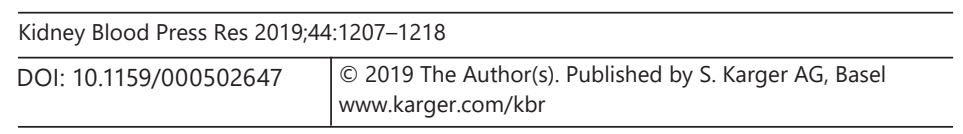

Marchelek-Myśliwiec et al.: FGF in CKD

\section{Determination of Biochemical Parameters}

Lipid concentrations (total cholesterol, high-density lipoprotein [HDL] and low-density lipoprotein [LDL] cholesterol and triacylglycerols) and glucose concentrations were determined in serum with an enzymatic method using the Architect c8000 analyser (ABBOTT, USA). GFR was estimated using the CKD-EPI equation.

\section{Determination of FGF21 and FGF19 Concentrations}

Serum FGF21 and FGF19 concentrations were measured using a commercially available ELISA kit (Human FGF 21 - Quantikine ELISA kit, R\&D System Minneapolis, MN, USA; Human FGF 19 - LSBio ELISA kit, LifeSpan BioSciences) according to the instructions of the manufacturer $[18,19]$.

\section{Determination of Body Composition}

Measurements of adipose tissue content (BF) and lean body mass (LM) were made using the dual X-ray absorptiometer method (Lunar Prodigy Advance, GE Healthcare).

\section{Statistical Analysis}

Non-parametric tests were used for statistical analysis of quantitative variables $(p<0.05$ for deviation from normal distribution; Shapiro-Wilk test). The Mann-Whitney U test was used for comparisons between 2 groups, and the Spearman's rank correlation coefficient was used to assess associations between variables within groups. The general linear model was used for multivariate analysis with logarithmically transformed FGF19 or FGF21 concentrations as dependent variables and age, sex and parameters significantly associated with FGF19 or FGF21 in univariate analyses as independent variables. Values of $p<0.05$ were considered statistically significant.

\section{Results}

\section{Baseline Characteristics}

The study groups (C, CKD, HD and Tx) did not differ significantly in age and BMI with the exception of the CKD group, which was significantly older and showed a higher BMI compared to Tx patients. However, there were significant differences in GFR between groups (Table 1).

\section{Concentrations of Biochemical Parameters}

Glucose levels were significantly higher in HD patients compared to the controls and patients after kidney transplantation. Serum total cholesterol levels were significantly higher in HD patients compared to the control group and patients with CKD (Table 1).

\section{Concentrations of FGF19 and FGF21}

FGF19 concentrations differed significantly between all groups (except HD vs. C). The highest serum concentration of FGF19 was observed in CKD patients and the lowest in the Tx group. Significantly higher serum FGF21 concentrations were found in patients in the CKD group compared to the C, HD and Tx groups (Table 1; Fig. 1, 2).

\section{Body Composition}

The study groups presented comparable body content; fat tissue and lean body mass did not differ significantly (Table 1). 
Fig. 1. Serum FGF19 concentrations in studied groups. * Serum FGF19 concentrations were higher in the CKD group ( $p=0.001)$ and lower in the Tx group ( $p=$ $0.039)$ than in the control. \# Serum FGF19 concentrations were lower in the HD ( $p=0.034)$ and Tx $(p<0.00001)$ groups compared to the CKD group. ^ Serum FGF19 concentrations were lower in the Tx group ( $p=0.000017)$ compared to the HD group. C, control group; CKD, patients with the CKD in stages 2-4 without haemodialysis; HD, haemodialysed patients with CKD; Tx, patients after a renal transplantation. FGF, fibroblast growth factors; GFR, glomerular filtration rate.

Fig. 2. Serum FGF21 concentrations in studied groups. * Serum FGF21 concentrations were higher in the CKD group ( $p=0.046$ ) than in the control. ${ }^{\#}$ Serum FGF21 concentrations were lower in the HD ( $p=0.0068)$ and Tx ( $p=0.019)$ groups compared to the CKD group. C, control group; CKD, patients with the chronic kidney disease in stages 2-4 without haemodialysis; HD, haemodialysed patients with CKD; Tx, patients after a renal transplantation; FGF, fibroblast growth factors.
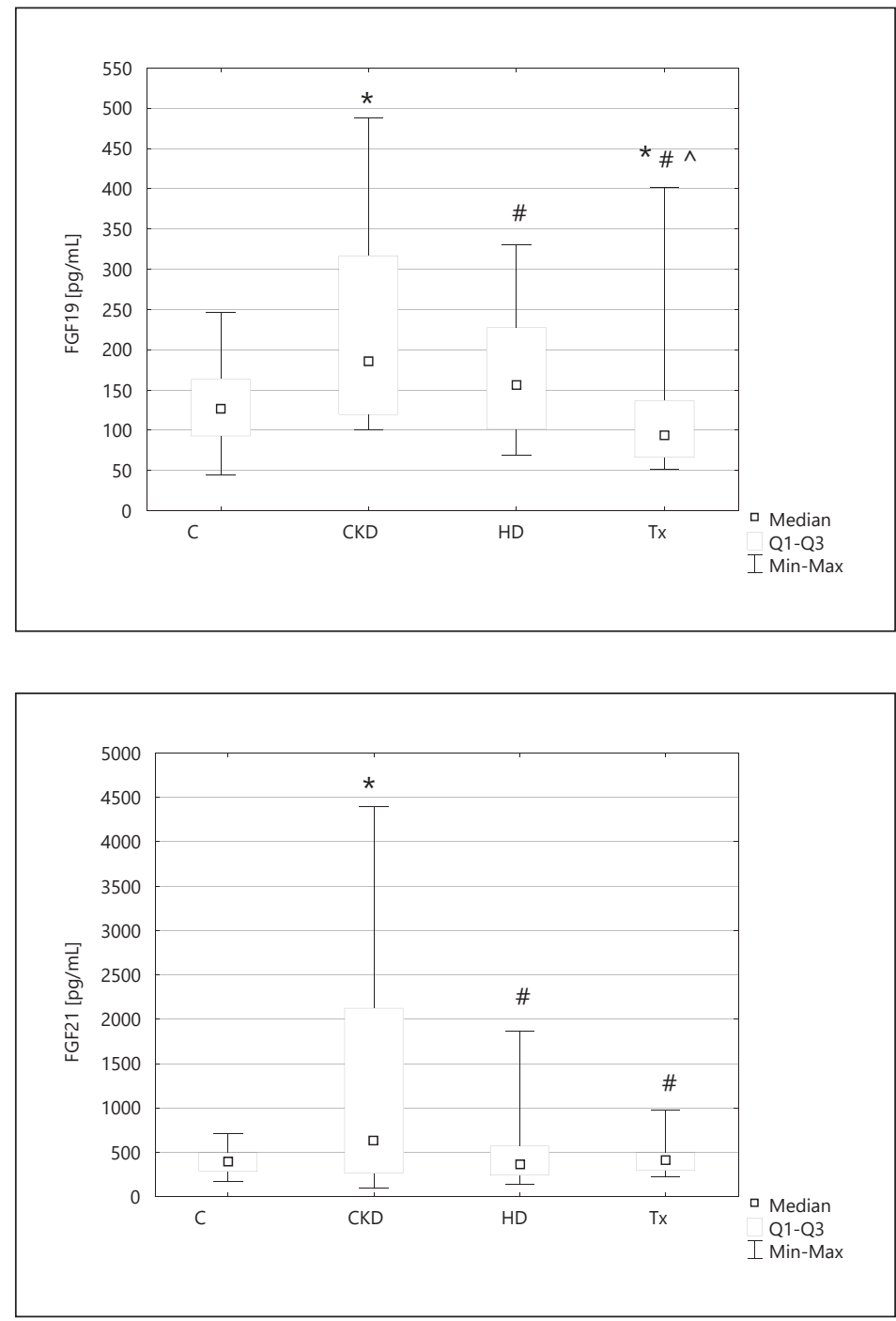

\section{Correlations between FGF19 and FGF21 and Clinical and Biochemical Parameters and} Body Composition

In the $\mathrm{C}$ group, the FGF19 concentration exhibited a negative correlation with glucose, as well as total and LDL cholesterol levels (Table 2). In the CKD group, we observed a negative correlation between the FGF19 concentration and GFR and no correlation between the FGF21 concentration and GFR (Table 2; Fig. 3, 4). The number of (CKD 2) patients was too low for separate analysis, so FGF21 and FGF19 concentrations were compared between (CKD $2+3$ ) patients (GFR $\geq 30$ ) and (CKD 4) patients (GFR <30). The FGF19 serum concentrations were significantly higher in stage 4 CKD patients compared to stage 2 and stage 3 CKD patients, combined $(295.9 \pm 118.5 \mathrm{pg} / \mathrm{mL}$ vs. $180.3 \pm 97.5 \mathrm{pg} / \mathrm{mL}$, respectively, $p=0.00020)$, consistently with a negative correlation between GFR and FGF19. There was no significant association between FGF21 serum concentrations and the stages of CKD.

In patients after kidney transplantation, there were negative correlations between FGF19 and GFR (Fig. 5), as well as HDL levels, and a positive correlation between FGF19 and triacylglycerols (Table 2). 
Kidney

Blood Pressure

Research

Fig. 3. Correlation between serum FGF19 concentration and GFR in CKD group. CKD, chronic kidney disease; GFR, glomerular filtration rate; FGF, fibroblast growth factors.

Fig. 4. Correlation between serum FGF21 concentration and GFR in CKD group. CKD, chronic kidney disease; GFR, glomerular filtration rate; FGF, fibroblast growth factors.

\begin{tabular}{l|l}
\hline Kidney Blood Press Res 2019;44:1207-1218 \\
\hline DOI: 10.1159/000502647 & $\begin{array}{l}\text { ○ 2019 The Author(s). Published by S. Karger AG, Basel } \\
\text { www.karger.com/kbr }\end{array}$ \\
\hline
\end{tabular}
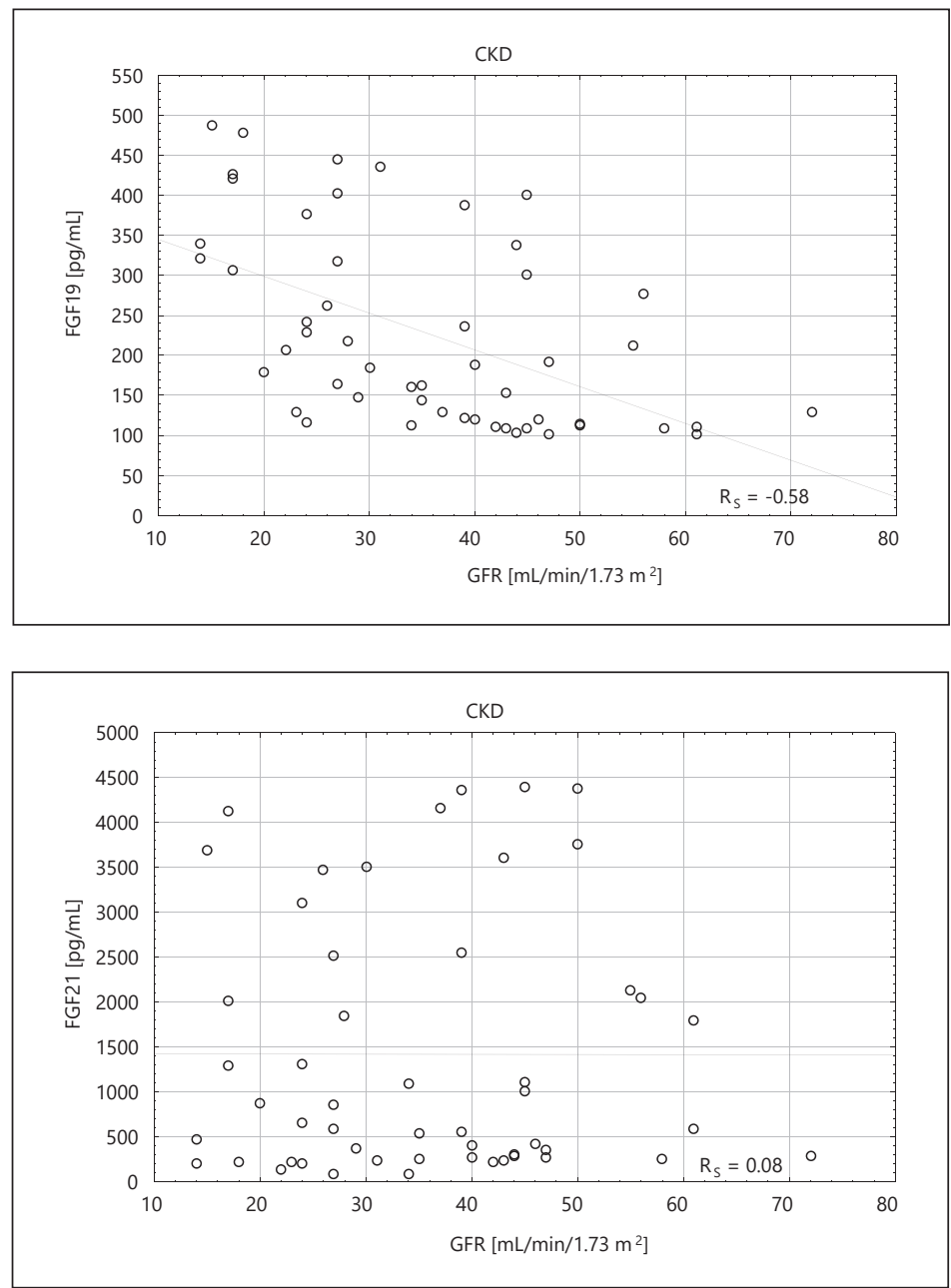

Table 2. The correlations of FGF19 and FGF21 ( $\left.\mathrm{R}_{\mathrm{s}}\right)$ with selected parameters

\begin{tabular}{|c|c|c|c|c|c|c|c|c|}
\hline \multirow[t]{2}{*}{ Parameters } & \multicolumn{4}{|l|}{ FGF19 } & \multicolumn{4}{|l|}{ FGF21 } \\
\hline & $\mathrm{C}$ & CKD & HD & $\mathrm{Tx}$ & $\mathrm{C}$ & CKD & HD & $\mathrm{Tx}$ \\
\hline Age (years) & -0.04 & 0.003 & 0.19 & 0.12 & 0.13 & 0.10 & -0.02 & 0.34 \\
\hline GFR & 0.003 & -0.58 & - & -0.67 & -0.41 & 0.08 & - & -0.48 \\
\hline BMI & -0.15 & 0.03 & -0.14 & 0.18 & 0.02 & 0.12 & 0.30 & 0.15 \\
\hline Glucose & -0.49 & 0.14 & -0.08 & 0.05 & -0.002 & 0.06 & 0.28 & 0.02 \\
\hline $\mathrm{CH}$ & -0.43 & -0.13 & -0.10 & 0.12 & 0.11 & -0.19 & 0.37 & 0.06 \\
\hline HDL & -0.12 & -0.07 & -0.06 & -0.35 & -0.12 & -0.09 & 0.22 & -0.02 \\
\hline LDL & -0.53 & -0.14 & 0.15 & 0.19 & 0.03 & -0.19 & 0.12 & 0.07 \\
\hline $\mathrm{TG}$ & 0.30 & 0.01 & -0.26 & 0.30 & 0.41 & 0.16 & 0.28 & 0.12 \\
\hline $\mathrm{BF}$ & -0.26 & 0.05 & -0.23 & -0.01 & -0.27 & -0.11 & 0.22 & 0.02 \\
\hline LM & 0.05 & -0.22 & -0.10 & 0.21 & -0.12 & 0.39 & 0.03 & 0.14 \\
\hline
\end{tabular}

$\mathrm{R}_{\mathrm{S}}$, Spearman rank coefficient (statistically significant results are marked bold; $p<0.05$ ). C, control group; CKD, patients with the chronic kidney disease in stages 2-4 without haemodialysis; HD, haemodialysed patients with CKD; Tx, patients after a renal transplantation; BF, body fat; BMI, body mass index; $\mathrm{CH}$, total cholesterol; GFR, glomerular filtration rate; HDL, high-density lipoprotein; LDL, low-density lipoprotein; LM, lean mass; TG, triacylglycerols; FGF, fibroblast growth factors. 
Fig. 5. Correlation between serum FGF19 concentration and GFR in Tx group. Tx, renal transplantation; GFR, glomerular filtration rate; FGF, fibroblast growth factors.

Fig. 6. Correlation between serum FGF21 concentration and GFR in Tx group. Tx, renal transplantation; GFR, glomerular filtration rate; FGF, fibroblast growth factors.
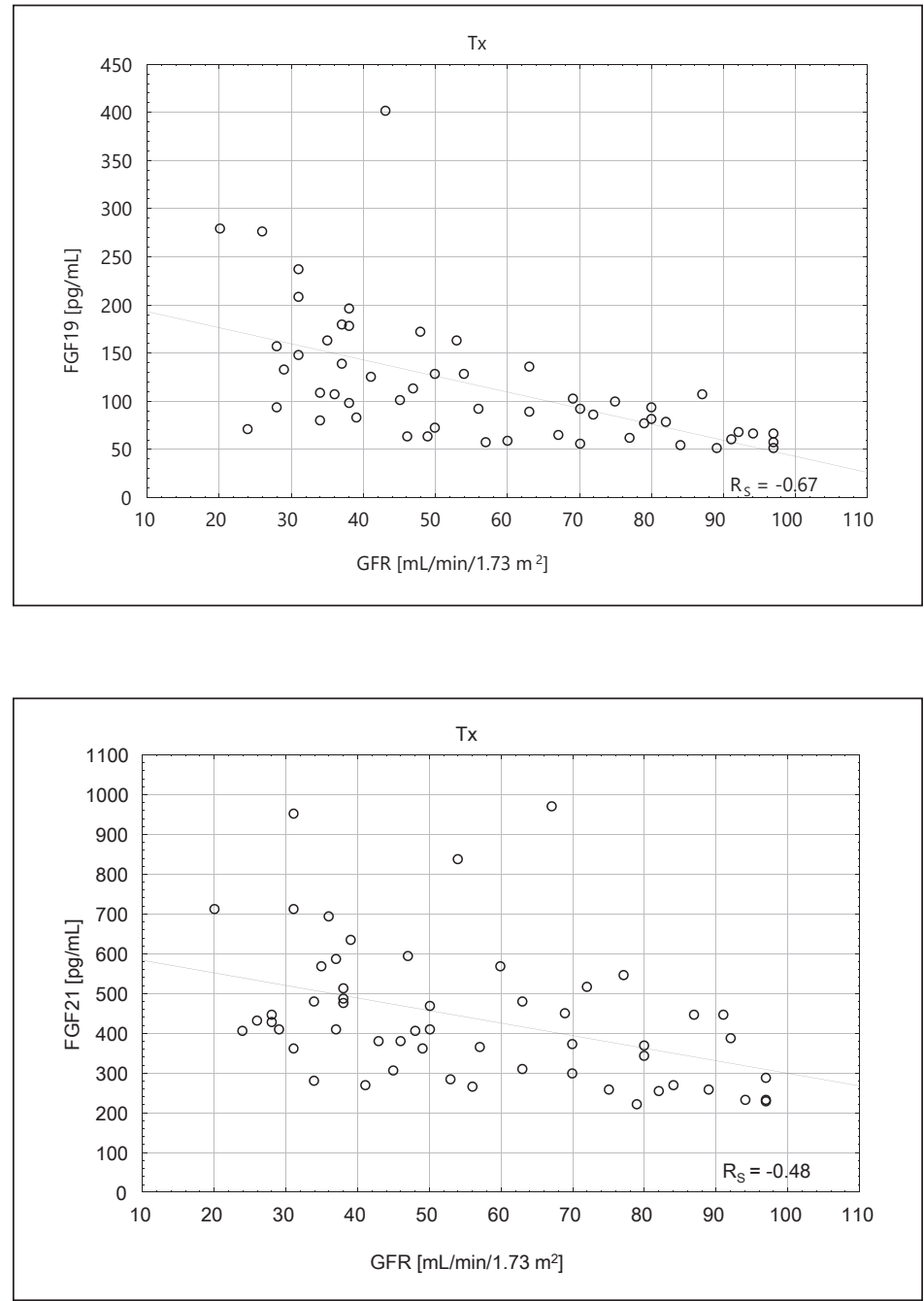

Negative correlations were found between serum FGF21 concentrations and GFR in patients after Tx (Fig. 6), while positive correlations were observed between FGF21 concentrations and lean body mass in the CKD group, BMI and total cholesterol in the HD patients and age in patients after Tx (Table 2).

In CKD group females compared to CKD group males, we observed significantly higher FGF19 concentrations $(259.2 \pm 120.5 \mathrm{pg} / \mathrm{mL}$ vs. $199.4 \pm 114.4 \mathrm{pg} / \mathrm{mL}$, respectively, $p=0.038)$, as well as significantly lower FGF21 serum concentrations $(803.4 \pm 1,095.6 \mathrm{pg} / \mathrm{mL}$ vs. $1,943.9$ $\pm 1,511.5 \mathrm{pg} / \mathrm{mL}$, respectively, $p=0.0022$ ). In females after kidney transplantation, FGF19 serum concentrations were significantly lower compared to that observed in males after $\mathrm{Tx}$ $(100.8 \pm 74.3 \mathrm{pg} / \mathrm{mL}$ vs. $126.2 \pm 61.7 \mathrm{pg} / \mathrm{mL}$, respectively, $p=0.032)$.

\section{Multivariate Analyses}

In the CKD group, being female and having lower GFR were independent predictors of higher FGF19 concentrations. The association of FGF21 with lean mass lost significance when gender was included in the multivariate model - it appears that the positive univariate correlation was caused by a positive association of being male with both higher lean mass and a higher FGF21 concentration. 


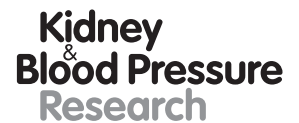

Research \begin{tabular}{l|l}
\hline Kidney Blood Press Res 2019;44:1207-1218 \\
\hline DOI: 10.1159/000502647 & $\begin{array}{l}\text { @ 2019 The Author(s). Published by S. Karger AG, Basel } \\
\text { www.karger.com/kbr }\end{array}$ \\
\hline
\end{tabular}

Marchelek-Myśliwiec et al.: FGF in CKD

In the HD group, higher total cholesterol was an independent predictor of higher FGF21, while a positive association with BMI was of borderline significance.

In the Tx group, a lower GFR was an independent predictor of both higher FGF19 and FGF21 concentrations, while the negative association of FGF19 with HDL cholesterol was of borderline significance.

\section{Discussion}

We examined FGF19 and FGF21 concentrations in patients with CKD who did not require haemodialysis, in HD patients with CKD and in patients with CKD after Tx. The highest FGF19 and FGF21 concentrations were observed in CKD patients without haemodialysis. Our results indicate that haemodialysis and transplantation cause the reduction of FGF19 and FGF21 concentrations in patients with CKD. Moreover, FGF19 and FGF21 concentrations were negatively correlated with GFR values.

FGF19 and FGF21 are relatively newly discovered proteins. Their significance in CKD is not yet known. Elevated FGF21 levels have been observed in both acute and chronic renal diseases [20,21]. The increase in FGF21 concentrations, along with CKD progression, can be explained as the body's defensive response to adverse metabolic disorders associated with kidney disease, such as insulin resistance or metabolic stress [14]. A study with 700 subjects found that increased FGF21 concentrations, independent of other factors, were associated with abnormal glucose metabolism and insulin resistance and were positively correlated with triacylglycerols and BMI [22]. In our study, in the HD group, there was a positive correlation between FGF21 and BMI and total cholesterol. Similarly, in the Tx group, the correlation between FGF21 and GFR was negative. Kidney disease is accompanied by insulin resistance and increased cardiovascular risk, which has also been observed in the obese population with metabolic syndrome, in which higher concentrations of FGF21 have been shown as well [23]. Our knowledge about the role of FGF21 in the kidneys is not complete. In our study, FGF21 levels were significantly higher in the CKD group compared to the HD group. In a study by Lin et al. [24], a linear increase in FGF21 concentrations, along with a decrease in GFR, was observed in CKD patients. The highest FGF21 concentrations were observed in patients with GFR below $30 \mathrm{~mL} / \mathrm{min}$. These changes in the metabolic profile, inflammation and loss of residual diuresis after the initiation of haemodialysis may induce the synthesis of FGF21.

As with FGF21, FGF19 concentrations in our study were highest in the CKD group. Both are involved in metabolic regulation and carbohydrate homeostasis. A study on HD patients found FGF19 concentrations to be 1.5 times higher compared to the control group [25]. The role of FGF19 in CKD patients is unknown. Animal studies conducted to date have focused on the metabolic activity of FGF19, and there are no data on FGF19 in renal failure. It is possible that, similarly to FGF21, the concentrations of FGF19 in kidney disease result from metabolic changes, inflammation and probably even nutrition.

The results of the present study have shown that haemodialysis and kidney transplantation reduce FGF19 and FGF21 concentrations. Previous studies suggest that factors present in ESRD, such as uremic toxins, metabolic acidosis, hyperphosphatemia, chronic inflammation and increased oxidative stress, insulin resistance and metabolic syndrome may contribute to the elevation of serum FGF19 and FGF21 levels in ESRD. Perhaps, in reducing the above factors, haemodialysis and kidney transplantation reduce FGF19 and FGF21 concentrations.

The pathophysiological significance of increased serum FGF19 and FGF21 concentrations in ESRD is not clear. In ESRD patients, it is not known whether the increased serum 


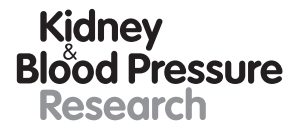

\begin{tabular}{l|l}
\hline Kidney Blood Press Res 2019;44:1207-1218 \\
\hline DOI: 10.1159/000502647 & $\begin{array}{l}\text { @ 2019 The Author(s). Published by S. Karger AG, Basel } \\
\text { www.karger.com/kbr }\end{array}$ \\
\hline
\end{tabular}

Marchelek-Myśliwiec et al.: FGF in CKD

concentrations of FGF19 and FGF21 are caused by disease factors or whether the increased concentrations are protective against the abovementioned factors. Increased serum FGF21 levels were associated with a high mortality rate in patients with ESRD [26]. In contrast, some clinical studies suggest that elevated serum FGF19 and FGF21 concentrations in ESRD are protective against glucotoxicity and lipotoxicity, oxidative stress and inflammation. In physiological conditions, the liver is the main source of FGF21; however, in ESRD, adipose tissue, skeletal muscle and kidney may be a source of FGF21. The disease factors in ESRD may increase FGF21 serum levels because ESRD is characterised by systemic and cellular metabolic stress, insulin resistance, metabolic syndrome and dyslipidaemia. Increased FGF21 secretion may augment glucose uptake by adipose tissue and muscles and re-establish metabolic balance. The increased FGF21 serum levels in ESRD can be explained not only by its decreased renal clearance but also by enhanced FGF21 secretion by skeletal muscle and adipose tissue.

There was a positive correlation between FGF21 and FGF23 and biochemical parameters, such as cholesterol, triacylglycerols, LDL cholesterol, fasting blood sugar, creatinine, HbA1c and urinary albumin excretion [27]. Lee et al. [28] showed that elevated serum FGF21 levels may be a useful biomarker for predicting kidney disease progression, especially in the early stages of diabetic nephropathy. The study by Li et al. [19] suggests that in haemodialysis patients with ESRD, the postprandial FGF19 response appears to be blunted, with partial normalisation following antioxidative treatments. A blunted FGF19 response was associated with impaired insulin and C-peptide signalling. Moreover, serum FGF-21 concentrations are associated with residual renal function and insulin resistance in ESRD patients receiving long-term peritoneal dialysis. Han et al. [29] and González et al. [30] have shown that FGF21 might be an important endocrine agent in peritoneal dialysis patients and could act as hormonal signalling to maintain glucose homeostasis and prevent potential insulin resistance. FGF21 might play a protective role against the development of insulin resistance over time in patients undergoing a continuous glucose load.

Rusu et al. [31] examined the correlation between FGF21 and tumour necrosis factor (TNF)-like weak inducer of apoptosis, as well as the correlation between FGF21 and endothelial dysfunction in HD patients. Increased FGF21 levels in HD patients correlated with atherosclerosis risk factors, hypercholesterolemia, hypertriglyceridemia, HA, increased levels of TNF-like weak inducer of apoptosis, as well as endothelial dysfunction [31]. There was also a positive correlation between FGF21 and the high-sensitivity C-reactive protein, as well as a negative correlation with renal function [32]. Maternal FGF21 serum concentrations are significantly increased in preeclampsia during pregnancy. Furthermore, triacylglycerols, GFR and LDL cholesterol are independent predictors of circulating FGF21 in pregnant women [33].

In our study, FGF21 and FGF19 did not correlate with the amount of adipose tissue. FGF21 only correlated with lean body mass in the CKD group. Previous studies have reported that FGF21 concentrations in obese patients were significantly higher than in the control group with a normal BMI [34]. Currently, FGF21 is recognised for having 2 important target sites, the central nervous system and adipose tissue. In addition, FGF21 exerts short- and long-term effects. A single administration of FGF21 leads to a rapid decline in glycaemia and improvement of insulin resistance, whereas cyclic long-term FGF21 administration causes a decrease in body weight [35]. There are no data on the correlation of FGF21 with adipose tissue in patients with kidney disease. FGF19 has been studied in obese patients undergoing body weight reduction procedures, in whom FGF19 concentrations were lower compared to the control group. In the same patients, FGF21 concentrations were elevated in comparison to the control group. The conclusion was that FGF19 is associated more with adipose tissue, while FGF21 is associated more with carbohydrate homeostasis [36]. The apparent lack of 
correlation of FGF19 and FGF21 with the amount of adipose tissue in our study may reflect characteristics of our study population; our subjects were not obese and the BMI in our groups did not differ significantly. In the control group only, there was a negative correlation between FGF19 and the glucose concentration; however, that does not exclude a potential influence of the $2 \mathrm{FGFs}$ on carbohydrate metabolism in CKD, HD and Tx patients.

Our results revealed increased FGF19 and FGF21 concentrations in patients with CKD who were not HD. Moreover, we found that haemodialysis and kidney transplantation caused the reduction of FGF19 and FGF21 concentrations in patients with CKD. Our results also suggest that increased concentrations of FGF19 and FGF21 in patients with CKD may be associated with the metabolism of lipids and carbohydrates. Nevertheless, the involvement of FGF19 and FGF21 in CKD requires further investigations.

\section{Disclosure Statement}

The authors report no conflicts of interest.

\section{References}

1 Vadakedath S, Kandi V. Dialysis: A review of the mechanisms underlying complications in the management of chronic renal failure. Cureus. 2017 Aug; 9(8):e1603.

2 Chalmers L, Kaskel FJ, Bamgbola O. The role of obesity and its bioclinical correlates in the progression of chronic kidney disease. Adv Chronic Kidney Dis. 2006 Oct;13(4):352-64.

3 Hsu CY, McCulloch CE, Iribarren C, Darbinian J, Go AS. Body mass index and risk for end-stage renal disease. Ann Intern Med. 2006 Jan;144(1):21-8.

4 Lu JL, Kalantar-Zadeh K, Ma JZ, Quarles LD, Kovesdy CP. Association of body mass index with outcomes in patients with CKD. J Am Soc Nephrol. 2014 Sep;25(9):2088-96.

5 Caetano C, Valente A, Oliveira T, Garagarza C. Body composition and mortality predictors in hemodialysis patients. J Ren Nutr. 2016 Mar;26(2):81-6.

6 Camilleri B, Bridson JM, Sharma A, Halawa A. From chronic kidney disease to kidney transplantation: the impact of obesity and its treatment modalities. Transplant Rev (Orlando). 2016 Oct;30(4):203-11.

7 Xiang DM, Song XZ, Zhou ZM, Liu Y, Dai XY, Huang XL, et al. Chronic kidney disease promotes chronic inflammation in visceral white adipose tissue. Am J Physiol Renal Physiol. 2017 Apr;312(4):F689-701.

8 Xu H, Huang X, Arnlöv J, Cederholm T, Stenvinkel P, Lindholm B, et al. Clinical correlates of insulin sensitivity and its association with mortality among men with CKD stages 3 and 4. Clin J Am Soc Nephrol. 2014 Apr; 9(4): 690-7.

9 Zhang F, Yu L, Lin X, Cheng P, He L, Li X, et al. Minireview: roles of fibroblast growth factors 19 and 21 in metabolic regulation and chronic diseases. Mol Endocrinol. 2015 Oct;29(10):1400-13.

10 Kurosu H, Choi M, Ogawa Y, Dickson AS, Goetz R, Eliseenkova AV, et al. Tissue-specific expression of betaKlotho and fibroblast growth factor (FGF) receptor isoforms determines metabolic activity of FGF19 and FGF21. J Biol Chem. 2007 Sep;282(37):26687-95.

11 Zhang C, Shao M, Yang H, Chen L, Yu L, Cong W, et al. Attenuation of hyperlipidemia- and diabetes-induced early-stage apoptosis and late-stage renal dysfunction via administration of fibroblast growth factor-21 is associated with suppression of renal inflammation. PLoS One. 2013 Dec;8(12):e82275.

12 Han X, Quarles LD. Multiple faces of fibroblast growth factor-23. Curr Opin Nephrol Hypertens. 2016 Jul;25(4): 333-42.

13 Badman MK, Pissios P, Kennedy AR, Koukos G, Flier JS, Maratos-Flier E. Hepatic fibroblast growth factor 21 is regulated by PPARalpha and is a key mediator of hepatic lipid metabolism in ketotic states. Cell Metab. 2007 Jun;5(6):426-37.

14 Kharitonenkov A, Shiyanova TL, Koester A, Ford AM, Micanovic R, Galbreath EJ, et al. FGF-21 as a novel metabolic regulator. J Clin Invest. 2005 Jun;115(6):1627-35.

15 Choi JR, Kim JY, Park IH, Huh JH, Kim KW, Cha SK, et al. Serum fibroblast growth factor 21 and new-onset metabolic syndrome: KoGES-ARIRANG study. Yonsei Med J. 2018 Mar;59(2):287-93.

16 Chiang JY. Bile acids: regulation of synthesis. J Lipid Res. 2009 Oct;50(10):1955-66.

17 Donate-Correa J, Martín-Núñez E, Delgado NP, de Fuentes MM, Arduan AO, Mora-Fernández C, et al. Implications of Fibroblast growth factor/Klotho system in glucose metabolism and diabetes. Cytokine Growth Factor Rev. 2016 Apr;28:71-7.

18 Choi HS, Lee HA, Kim SW, Cho EH. Association between Serum Fibroblast Growth Factor 21 Levels and Bone Mineral Density in Postmenopausal Women. Endocrinol Metab (Seoul). 2018 Jun;33(2):273-7. 
19 Li M, Qureshi AR, Ellis E, Axelsson J. Impaired postprandial fibroblast growth factor (FGF)-19 response in patients with stage 5 chronic kidney diseases is ameliorated following antioxidative therapy. Nephrol Dial Transplant. 2013 Nov;28 Suppl 4:iv212-9.

20 Stein S, Bachmann A, Lössner U, Kratzsch J, Blüher M, Stumvoll M, et al. Serum levels of the adipokine FGF21 depend on renal function. Diabetes Care. 2009 Jan;32(1):126-8.

21 Hindricks J, Ebert T, Bachmann A, Kralisch S, Lössner U, Kratzsch J, et al. Serum levels of fibroblast growth factor-21 are increased in chronic and acute renal dysfunction. Clin Endocrinol (Oxf). 2014 Jun;80(6):918-24.

22 Semba RD, Sun K, Egan JM, Crasto C, Carlson OD, Ferrucci L. Relationship of serum fibroblast growth factor 21 with abnormal glucose metabolism and insulin resistance: the Baltimore Longitudinal Study of Aging. J Clin Endocrinol Metab. 2012 Apr;97(4):1375-82.

23 Zhang X, Yeung DC, Karpisek M, Stejskal D, Zhou ZG, Liu F, et al. Serum FGF21 levels are increased in obesity and are independently associated with the metabolic syndrome in humans. Diabetes. 2008 May;57(5):124653.

24 Lin Z, Zhou Z, Liu Y, Gong Q, Yan X, Xiao J, et al. Circulating FGF21 levels are progressively increased from the early to end stages of chronic kidney diseases and are associated with renal function in Chinese. PLoS One. 2011 Apr;6(4):e18398.

25 Reiche M, Bachmann A, Lössner U, Blüher M, Stumvoll M, Fasshauer M. Fibroblast growth factor 19 serum levels: relation to renal function and metabolic parameters. Horm Metab Res. 2010 Mar;42(3):178-81.

26 Kohara M, Masuda T, Shiizaki K, Akimoto T, Watanabe Y, Honma S, et al. Association between circulating fibroblast growth factor 21 and mortality in end-stage renal disease. PLoS One. 2017 Jun;12(6):e0178971.

27 El-Saeed AM, El-Mohasseb GF. Circulating Fibroblast Growth Factors 21 and 23 as Biomarkers of Progression in Diabetic Nephropathy in Type 2 Diabetes with Normoalbuminuria. Egypt J Immunol. 2017 Jun;24(2):93-9.

28 Lee CH, Hui EY, Woo YC, Yeung CY, Chow WS, Yuen MM, et al. Circulating fibroblast growth factor 21 levels predict progressive kidney disease in subjects with type 2 diabetes and normoalbuminuria. J Clin Endocrinol Metab. 2015 Apr;100(4):1368-75.

29 Han SH, Choi SH, Cho BJ, Lee Y, Lim S, Park YJ, et al. Serum fibroblast growth factor-21 concentration is associated with residual renal function and insulin resistance in end-stage renal disease patients receiving longterm peritoneal dialysis. Metabolism. 2010 Nov;59(11):1656-62.

30 González E, Díez JJ, Bajo MA, del Peso G, Grande C, Rodríguez O, et al. Fibroblast Growth Factor 21 (FGF-21) in Peritoneal Dialysis Patients: Natural History and Metabolic Implications. PLoS One. 2016 Mar;11(3):e0151698.

31 Rusu CC, Racasan S, Kacso IM, Moldovan D, Potra A, Tirinescu D, et al. The metabolic hormone FGF21 is associated with endothelial dysfunction in hemodialysis patients. Int Urol Nephrol. 2017 Mar;49(3):517-23.

32 Trakarnvanich T, Prommool S, Kurathong S, Teepprasan T, Wang Y. Associations Among Cardio-Ankle Vascular Index, Carotid Intima-Media Thickness, and Fibroblast Growth Factor-21 Levels in Kidney Transplant Patients. Transplant Proc. 2017 Oct;49(8):1791-6.

33 Stepan H, Kley K, Hindricks J, Kralisch S, Jank A, Schaarschmidt W, et al. Serum levels of the adipokine fibroblast growth factor-21 are increased in preeclampsia. Cytokine. 2013 May;62(2):322-6.

34 Crujeiras AB, Gomez-Arbelaez D, Zulet MA, Carreira MC, Sajoux I, de Luis D, et al. Plasma FGF21 levels in obese patients undergoing energy-restricted diets or bariatric surgery: a marker of metabolic stress? Int J Obes. 2017 Oct;41(10):1570-8.

35 BonDurant LD, Ameka M, Naber MC, Markan KR, Idiga SO, Acevedo MR, et al. FGF21 regulates metabolism through adipose-dependent and -independent mechanisms. Cell Metab. 2017 Apr;25(4):935-944.e4.

36 Gómez-Ambrosi J, Gallego-Escuredo JM, Catalán V, Rodríguez A, Domingo P, Moncada R, et al. FGF19 and FGF21 serum concentrations in human obesity and type 2 diabetes behave differently after diet- or surgicallyinduced weight loss. Clin Nutr. 2017 Jun;36(3):861-8. 